\title{
Tattoo Site
}

National Cancer Institute

\section{Source}

National Cancer Institute. Tattoo Site. NCI Thesaurus. Code C77684.

The anatomic site at which a tattoo is present. 